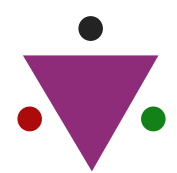

IJCRR

Section: Healthcare

Sci. Journal Impact

Factor: $6.1(2018)$

ICV: 90.90 (2018)

(c) (i) (8)

Copyright@IJCRR

\section{Comparative Study of Visual Acuity and Astigmatism Determination Between Small Incision Cataract Surgery and Phacoemulsification by Corneal Topography}

\author{
Neha Bajpayee ${ }^{1}$, Pravin K. Tidake ${ }^{2}$, Prasanna M. Palsodkar ${ }^{3}$ \\ Junior Resident, Department of Ophthalmology, Jawaharlal Nehru Medical College, Datta Meghe Institute of Medical Sciences (Deemed \\ University), Sawangi (Meghe), Wardha, Maharashtra, India; 'Professor, Department of Ophthalmology, Jawaharlal Nehru Medical College, \\ Datta Meghe Institute of Medical Sciences (Deemed University), Sawangi (Meghe), Wardha, Maharashtra, India; ${ }^{3}$ rofessor, Electronics \\ Engineering, Yeshwantrao Chavan College of Engineering, Nagpur, Maharashtra, India.
}

\title{
ABSTRACT
}

Background: Among adults as cataract being the most common cause of the correctable diminution of vision. There is 12.5 million blind, in India and blindness due to cataract is estimated to be $50 \%$ to $80 \%$. Due to cataract, an additional 3.8 million become blind each year in addition to backlog against which cataract surgeries are done every year are 2.7 million. Two main approaches are there for cataract extraction 1)manual small incision cataract extraction. 2)phacoemulsification. Corneal astigmatism is a type of refractive error where point focus is not formed leading to unequal refraction. This plays an important role in compliance with post-cataract extraction surgery. Since the cornea is responsible for $70 \%$ of eyes refractive power, its examination by corneal topography is of clinical importance in determining the quality of vision.

Objectives: 1) To study visual acuity changes between sics and phacoemulsification, 2) To study mean astigmatism between phacoemulsification and SICS.3)To estimate the net induced astigmatism and its association with refraction between SICS and phacoemulsification.

Methods: A observational interventional study with 120 patients will be conducted in a hospital based setting utilizing the data of patients eye. Preoperative visual acuity, astigmatism and postoperative visual acuity and corneal astigmatism are compared in two groups.

Expected Results: As compared to other studies present we expect to find to have a better visual outcome, the reduction is astigmatism by 6 weeks and less surgically induced astigmatism in the phacoemulsification group than Sics.

Key Words: Small Incision Cataract Surgery, Phacoemulsification, Surgically Induced Astigmatism, Visual Acuity, Corneal topography

\section{INTRODUCTION}

A cataract is the most common cause of the correctable diminution of vision in adults which leads to millions of individual going blind. ${ }^{1,2}$ Thus, by doing cataract surgeries morbidity of blindness can be reduced. Two major surgical approaches that have been followed are manual small incision cataract surgery, variant of ECCE and address high volume surgical outcome in dense cataracts, the procedure being by making self-sealing sclera-cornel tunnel. ${ }^{1-3}$ Another variant is phacoemulsification: removal of the nuclear portion of a cataractous lens using an aspi- rating and vibrating ultrasonic handpiece (that is phaco probe) ${ }^{4}$

Astigmatism refers to as refractive error in which parallel rays coming from infinity does not focus at one focal point but different meridians when accommodation is at the lens. ${ }^{3}$ Change in the curvature of cornea and lens causes changes in astigmatism. Compliance cataract surgery depends on corneal astigmatism after cataract surgery. ${ }^{2}$ Determination of astigmatism by photokertoscopy or videokeratoscopy as it allows mapping of the surface of the cornea and outer structure of eyes. ${ }^{2}$ As the cornea is responsible for $70 \%$ refractive

\section{Corresponding Author:}

Dr. Neha Bajpayee, Junior Resident, Department of Ophthalmology, Jawaharlal Nehru Medical College, Datta Meghe Institute of Medical Sciences (Deemed University), Sawangi (Meghe), Wardha, Maharashtra, India; Contact: 799943221; Email: nehabajpayee1601@gmail.com

ISSN: 2231-2196 (Print)

Received: 14.09 .2020
ISSN: 0975-5241 (Online)

Revised: 19.10 .2020
Accepted: 06.11 .2020
Published: 30.11 .2020 
power using corneal topography is important nowadays for determining the quality of vision. ${ }^{3}$ Since the evaluation of refractive changes in peripheral cornea and irregularities in the central cornea is not revealed by old keratometer, use of corneal topography gives an edge on a detailed examination of cornea and which can be used for improving the outcome of patients.

\section{OBJECTIVES}

- To study visual acuity changes between SICS AND PHACOEMULSIFICATION ${ }^{4}$.

- To study mean astigmatism between phacoemulsification and SICS

- To estimate the net induced astigmatism and its association with refraction between SICS and phacoemulsification.

\section{MATERIALS AND METHODS}

Study design: This is a prospective interventional study.

Setting: The study shall be conducted for two tears from September 2018 to September 2020. Patients presenting with cataract to the Out Patient Department of Acharya Vinoba Bhave Rural Hospital will be selected sequentially for the study after considering the inclusion and exclusion criteria. All data will be collected by a single experienced ophthalmologist and surgeries are performed by a single ophthalmic surgeon. The data will be collected in the form of a questionnaire to be filled by the investigator.

Participants: The participants will be sequentially selected on an OPD basis. Following criteria will be utilized. Study of 120 consecutive patients (randomly selected from eye OPD) assigned to undergo phacoemulsification (60 cases) and manual small incision cataract surgery ${ }^{5-8}$ (60 cases).

\section{Inclusion criteria}

- $\quad$ Patients aged more than 18 years.

- Randomly selected cataract patients coming to Eye OPD having different types and grades of cataract.

- Patients with nuclear sclerosis Grade 1/2/3 are selected.

- $\quad$ Patients will be selected irrespective of sex.

\section{Exclusion criteria}

- $\quad$ Patients equal to and more than grade 4 nuclear sclerosis.

- Patient less than 18 years.

- Paediatric cataract.

- Patients with coexisting glaucoma.

- Uveitis.

- Subluxated lens.

- Posterior segment pathology.

- Aphakic patients.
- Keratoconus.

- Corneal pathology.

- Connective tissue disorders.

- Traumatic cataract.

\section{Variables}

The variables to be studied are sex of the patient, age of the patient. Visual acuity, astigmatism preoperatively and visual acuity and surgically induced astigmatism ${ }^{9}$ postoperatively in both Sics and phacoemulsification group. ${ }^{2,48} \mathrm{~A}$ difference between the visual acuity postoperatively at $1^{\text {st }}$ day, $3^{\text {rd }}$ week and $6^{\text {th }}$ week is taken in both Sics and phacoemulsification group and as well as the difference in astigmatism preoperatively and postoperatively is assessed similarly in both the groups and will be calculated as a percentage for statistical analysis. The difference in visual acuity and surgically induces astigmatism will be compared between Sics and phacoemulsification group.

\section{Data sources/measurement}

The study will adhere to the tenets of the Declaration of Helsinki ${ }^{1}$, and it will be approved by an institutional ethics committee of DMIMSU.

Informed consent will be obtained from all subjects after the nature and possible consequences of the study will be explained to them. A comprehensive ophthalmic examination, including best-corrected visual acuity, slit-lamp examination measurement and fundus examination, will be performed on all participants. The mean corneal power (KM) and corneal astigmatism $(\mathrm{AST})^{10}$ in the $3.00 \mathrm{~mm}$ central zone will be evaluated for each patient by using a Corneal Topographer.

Participants undergo the choice of their surgery.

\section{MANUAL SMALL INCISION CATARACT SURGERY TECHNIQUE:}

After bridle suture, the conjunctival flap is taken then, the superior tunnel is made $6 \mathrm{~mm}$ length straight or frown incision on the sclera 1.5 to $2 \mathrm{~mm}$ away from the limbus. After capsulorhexis and hydro-dissection nucleus are delivered, cortex aspirated by symcoe irrigation and aspiration cannula and the intraocular lens is implanted. ${ }^{11}$

\section{PHACOEMULSIFICATION SURGICAL TECH- NIQUES:}

The superior clear corneal incision $(2.8 \mathrm{~mm})^{4,8}$ given just anterior to the limbus is given. After capsulorhexis did after making side port by 2.6 keratome $^{8}$ and hydro-dissection nucleus is delivered, cortex aspirated by symcoe irrigation and aspiration cannula and the foldable intraocular lens is implanted.

A detailed post-operative examination of patients is done on 1st day, 3weeks and 6 weeks. Examination including check- 
ing visual acuity, keratometry ${ }^{2}$, ophthalmoscopy( direct, indirect and slit-lamp biomicroscopy) is done.

Corneal Topography ${ }^{12}$ for keratometry and net induced astigmatism ${ }^{1,2}$ (the difference between postoperative astigmatism and preoperative astigmatism) will be done. At 6 weeks spectacles will be provided following final best-corrected visual acuity. All changes in corneal topography ${ }^{18}$ readings are recorded and tabulated for each corresponding follow up period.

\section{Study size}

Using sample size formula with the desired error of margin

$$
\mathrm{n}=\mathrm{Z}^{2} \alpha_{/ 2} * \mathrm{P} *(1-\mathrm{P})
$$

$\mathrm{d}^{2}$

Where

$\mathrm{Z}_{\alpha / 2}$ is the level of significance at $5 \%$,

Ie: $95 \%$ confidence interval $=1.96$

$\mathrm{P}=$ Prevalence of cataract $=7.39 \%$

$\mathrm{D}=$ Desired error of margin $=7 \%=0.07$

$\mathrm{n}=1.96^{2} \underline{x} \underline{0.0739 \times(1-0.0739)}$

$0.07^{2}$

$=53.65$

$=60$ patients needed in each group

\section{Sample size}

A minimum of 120 patients ( 60 eyes of 60 patients of Phacoemulsification and 60 eyes of 60 patients of Small Incision Cataract Surgery).

\section{Statistical methods}

Statistical analysis will be done using student t-test and Chisquare test, and values will be considered significant when $p$ $<0.005$. All data analysis will be performed using the SPSS statistical program.

\section{EXPECTED OUTCOMES/RESULTS}

Based on previous studies we expect to find surgically induced corneal astigmatism significantly less in microincision surgery (MICS) group than small-incision surgery (SICS) group, 1 week postoperatively and subsequently. Corresponding to the incision focal flattening and steeping around the flattened area is expected in photokeratoscopy or videokeratoscopy 2 days postoperatively in both groups. ${ }^{2}$ Reduction of flattening and steepening gradually to the preoperative shape by 6 weeks and these changes are expected significantly less in the phacoemulsification group than in the SICS group.

\section{DISCUSSION}

A statistically significant decrease in surgically induced astigmatism in the phacoemulsification group subsequently followed till $6^{\text {th }}$ week postoperatively than in SICS group..$^{15} \mathrm{~A}$ significant improvement in visual acuity in the phacoemulsification group than the SICS group by the end of $6^{\text {th }}$ week. Patients undergoing phacoemulsification also found to have less postoperative complication than who underwent SICS. ${ }^{7}$

Senile cataract is the main cause of the diminution of vision worldwide. The patient undergoing cataract surgery does not accept to wear spectacles after surgery and expects a clear vision. For this surgically induced astigmatism has to be reduced. Modern surgical technique and detailed evaluation of cornea before surgery aim to reduce surgically induce astigmatism. ${ }^{1}$ Two major factors affecting the induced astigmatism are length and site of the incision. The change in corneal curvature can lead to SIA and the astigmatic refractive error. Uncorrected astigmatism may result in blurred images and glare which can make patient uncomfortable and dissatisfied even with uneventful cataract surgery. ${ }^{11}$

Rapid changes in astigmatism with large changes in direction of the axis during the first postoperative month on photokeratometric measurements. ${ }^{12}$ Predomination of against-the-rule astigmatism is seen. Patients who underwent phacoemulsification showed small changes in corneal curvature a which is due to small incision size. ${ }^{1}$ Comparison was done between following conventional extracapsular cataract extraction (ECCE) with corneal incision of $10 \mathrm{~mm}$ and scleral incision after phacoemulsification of $5 \mathrm{~mm}$, results were significantly better than those following ECCE. ${ }^{13}$ In all postoperative time intervals, less astigmatism was induced by phacoemulsification than extracapsular surgery. ${ }^{2}$ Some of the studies on Cataract Surgery and Phacoemulsification have been reported. ${ }^{12-14}$

To study the effect of surgically ${ }^{13}$ induced astigmatism ${ }^{2,13}$ by two different surgeries for cataract extraction sample size requirement is large for a further basis. Different incision size and different incision site also lead to change in corneal astigmatism. Study with different size of incision other than $6 \mathrm{~mm}$ sclera tunnel incision in SICS and $2.8 \mathrm{~mm}^{8}$ superior clear corneal incision in phacoemulsification is required. ${ }^{4,8}$ Corneal topography can be used for manipulating incisions while doing cataract surgery in patients who has irregular cornea preoperatively, hence can help in providing better visual outcome later. ${ }^{4}$

\section{Interpretation:}

The difference in astigmatism in SICS and phacoemulsification group is due to the technique used in phacoemulsification cause small curvatural change in cornea postoperatively due to small incision size than in SICS group. ${ }^{4,7,13}$ Since inci- 
sion site and size in both groups have been the same throughout the study larger scale of study involving larger population with different size and site of the incision can be carried out for further validation of this study.

\section{CONCLUSION}

Review of all studies suggests that phacoemulsification causes less surgically induced astigmatism and better visual outcome.

\section{REFERENCES}

1. Harakuni U, Bubanale S, Smitha KS, Tenagi AL, Kshama KK, Meena A, et al. Comparison of surgically induced astigmatism with small incision cataract surgery and phacoemulsification. J Evol Med Dent Sci 2015;4(71):12354-61.

2. Yao K, Tang X, Ye P. Corneal astigmatism, high order aberrations, and optical quality after cataract surgery: microincision versus small incision. J Refract Surg 2006;22(11):S1079-82.

3. Klyce SD. Computer-assisted corneal topography. High-resolution graphic presentation and analysis of keratoscopy. Investig Ophthalmol Vis Sci 1984;25(12):1426-35.

4. Singhal S, Baheti A, Singh K, Acharya S, Acharya N. Cortical blindness in posterior reversible encephalopathy syndrome in postpartum eclampsia. Indian J Med Specialit 2019;10(4):229.

5. Kalode V, Daigavane S. Study of visual outcome and complications of iris-claw intraocular lens implantation to correct aphakia. J Datta Meghe Inst Med Sci Uni 2019;14(3):141.
6. Minassian DC, Rosen P, Dart JK, Reidy A, Desai P, Sidhu M. Extracapsular cataract extraction compared with small incision surgery by phacoemulsification: a randomised trial. Br J Ophthalmol 2001;85(7):822-9.

7. Vass C, Menapace R. Computerized statistical analysis of corneal topography for the evaluation of changes in corneal shape after surgery. Am J Ophthalmol 1994;118(2):177-84.

8. Olson RJ, Crandall AS. Prospective randomized comparison of phacoemulsification cataract surgery with a $3.2-\mathrm{mm}$ vs a 5.5 mm sutureless incision. Am J Ophthalmol 1998;125(5):612-20.

9. Neilsen PJ. Prospective evaluation of surgically induced astigmatism and astigmatic keratotomy effect of various small selfsealing incision. J Cataract Refract Surg 1995;21:43-8.

10. Uusitalo RJ, Tarkkanen A. Outcomes of small incision cataract surgery. J Cataract Refract Surg 1998;24(2):212-21.

11. Kohnen T, Dick B, Jacobi KW. Comparison of the induced astigmatism after temporal clear corneal tunnel incisions of different sizes. J Cataract Refract Surg 1995;21(4):417-24.

12. Shende V, Iratwar S, Daigavane S. Ocular manifestations in patients with intracranial space-occupying lesions. J Datta Meghe Inst Med Sci Uni 2019;14(3):119.

13. Muley S, Saoji C, Daigavane S. The Effect of Intracameral Adrenaline Bolus Injection on Pupil Size, Pulse Rate and Blood Pressure During Small Incision Cataract Surgery. Int J Cur Res Rev 2020; 12(14):35-42.

14. Muley S, Saoji C, Daigavane S, Patil R, Ghogare AS. Anxiety in Cataract Patients, Benefits of Counseling on Patient Anxiety in Routine Cataract Surgery. Int J Res Pharma Sci 2020;11(2):2620-5. 